\title{
Load Characteristics of Electric System for Distributing Power on Locality Based Criterion
}

\author{
Pawan Kumar
}

\begin{abstract}
The goal of this paper is to determine the load characteristics of Electric System for Distributing Power on Locality Based Criterion. In this paper we are determining how the value of load changes when there is a change in the value of Power Coefficients i.e. we are obtaining the variation of Active Power by varying the value of Kpv. The variation of Kpv by showing the variation in proportional value, Load profile and Voltage profile. The purpose of this paper is to show how a constant ZIP model shows variation when more than one such load is connected on that particular node. Because of change in the Kpv at a particular node there is a change in the load at that node. In this paper we have also show how the value of the power Coefficients varies by varying the load, voltage etc on that node. As power systems are designed and operated with less stability margin, the importance of good models and good data increases. Industry standard models facilitate the validation and certification of simulation software. In this paper we are using MATLAB software.
\end{abstract}

\section{INTRODUCTION}

$\mathrm{T}$ HE main objective of our project work is to determine how the load at a particular value changes at a particular node and these variations can be obtained by varying various parameters of a constant ZIP model. Suppose we have a Constant Impedence model at a node so if we are calculating the value of Power Coefficient i.e. Kpv then the value at that point will be 2 but if we connect more than one such load then the value of $\mathrm{Kpv}$ at that point will not be 2 but will differ from the original value due to which the value of load at that particular value will change.

Here we are only taking into consideration the variation of

- Variation in Load Profile

- Variation of Voltage Profile

- Variation of Power Proportion

The power-flow study, also known as load-flow study, is an important tool involving numerical analysis applied to a power system. A power-flow study usually uses simplified notation such as a one-line diagram and per-unit system, and focuses on various forms of AC power (i.e.: voltages, voltage angles, real power and reactive power).

Pawan Kumar

DOI: $10.9756 /$ BIJPSIC.10335

\section{Load Flow}

A load flow study is especially valuable for a system with multiple load centers such a refinery complex. The power flow study is an analysis of the system's capability to adequately supply the connected load. The total system losses, as well as individual line losses, also are tabulated. Transformer tap positions are selected to insure the correct voltage at critical locations such as motor control centers.

\section{Dynamic Stability}

System stability study is essential when adding, upgrading or evaluating existing generators within the facility. This study will evaluate overcurrent relay settings and or modifications to the protection scheme associated with the generators and utility. This time-based analysis will determine relay settings that will allow the generator out-of-step protection and over current protections to operate for a disturbance prior to loss of system stability and damage to equipment's. In general, load shedding (LS) can be defined as the amount of load that must nearly instantly be removed from a power system to keep the remaining portion of the system operational. This load reduction is in response to a system disturbance that results in an unbalanced condition of the amount of system load exceeding the available electric generation. Common disturbances that can cause this condition to occur include faults, loss of generation, switching errors, lightning strikes, etc.

\section{BACKGROUND OF PROBLEMS}

Electric utility analysts and their management require evidence of the benefits of improved load representation in order to justify the effort and expense of collecting and processing load data and, perhaps, modifying computer program load models. The benefits of improved load representation fall into the following categories:

\section{A. If Present Load Representation Produces Overly- Pessimistic Results}

1. In planning studies, the benefits of improved modeling will be in deferring or avoiding the expense of system modifications and equipment additions,

2. In operating studies, the benefits will be in increasing power transfer limits, with resulting economic benefits.

B. If Present Load Representation Produces Overly-Optimistic Results

1. In planning studies, the benefit of improved modeling will be in avoiding system inadequacies that may result in costly operating limitations, 
2. In operating studies, the benefit may be in preventing system emergencies resulting from overly-optimistic operating limits.

\section{Methodology OF WORK}

In our project we the showing that how the load at a particular Node changes as we know that in a constant ZIP model when we consider a load at any node then it could be either a constant power, constant current and constant impedence i.e. it will be having its $\mathrm{Kpv}$ either 0,1 or 2 but when more than one such load is connected at that node then the value of Kpv and Kqv which are related to active and reactive power respectively changes from its basic value. Now here we are showing the variations in

- Load Profile

- Power Profile

- Proportional Power

$$
\begin{aligned}
& P=P_{n}\left(P_{1}\left(\frac{V}{V n}\right)^{2}+P_{2} \frac{V}{V_{n}}+P_{3}\right), \\
& Q=Q_{n}\left(q_{1}\left(\frac{V}{V n}\right)^{2}+q_{2} \frac{V}{V_{n}}+q_{3}\right), \\
& P=P_{n}\left(\frac{V}{V_{n}}\right)^{K_{p v}}, Q=Q_{n}\left(\frac{V}{V_{n}}\right)^{k_{q v}}
\end{aligned}
$$

As we can see from the equation of Active Power that there can only be variation in load, power and proportional values we are considering that the frequency to be constant throughout the system.

\section{i. $\quad$ Variation in Load Profile}

In the load profile variation the value of load changes there is no change in the power and there is a change in the load and due to this variation there is change in the value of $\mathrm{Kpv}$ for Active Power and Kqv for Reactive Power due to which there is a change in the characteristic load at that node. This variation can be shown with the help of a graph which is drawn with the the help of Matlab software.
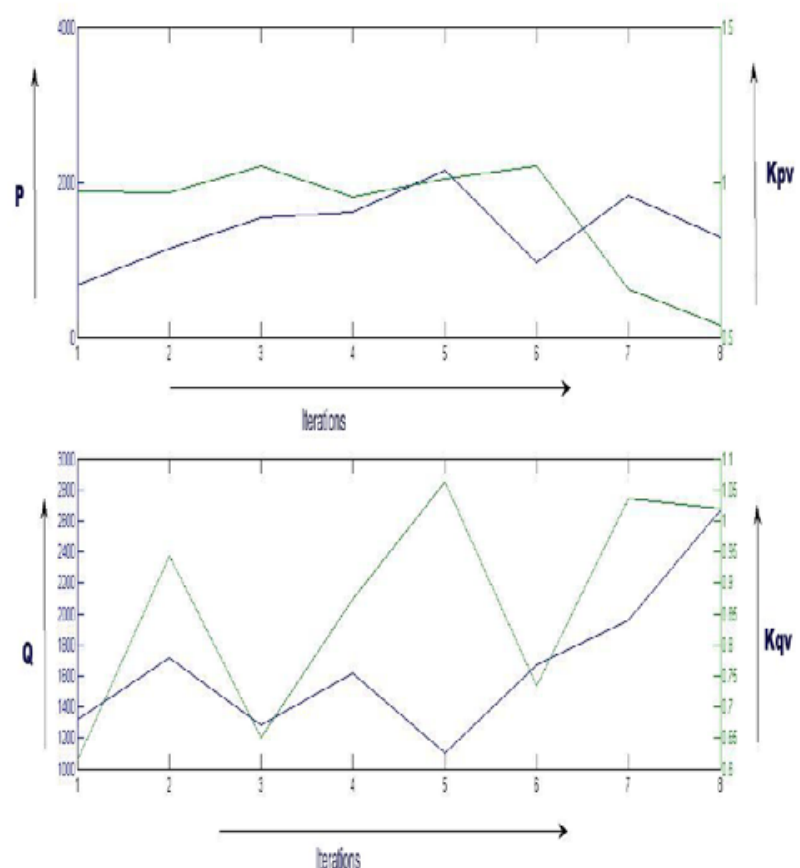

In the above graph we have shown the variation of Kpv and Composite load when there is a change in the load.

\section{ii. Variation in Voltage Profile}

In the voltage profile variation the value of load changes there is no change in the power and there is a constant fractional value i.e. the value of $P_{1}, P_{2}, P_{3}$ are constant and

their sum is always equal to unity ,due to this variation their is change in the value of Kpv for Active Power and Kqv for Reactive Power due to which there is a change in the characteristic load at that node. This variation can be shown with the help of a graph which is drawn with the the help of Matlab software. 

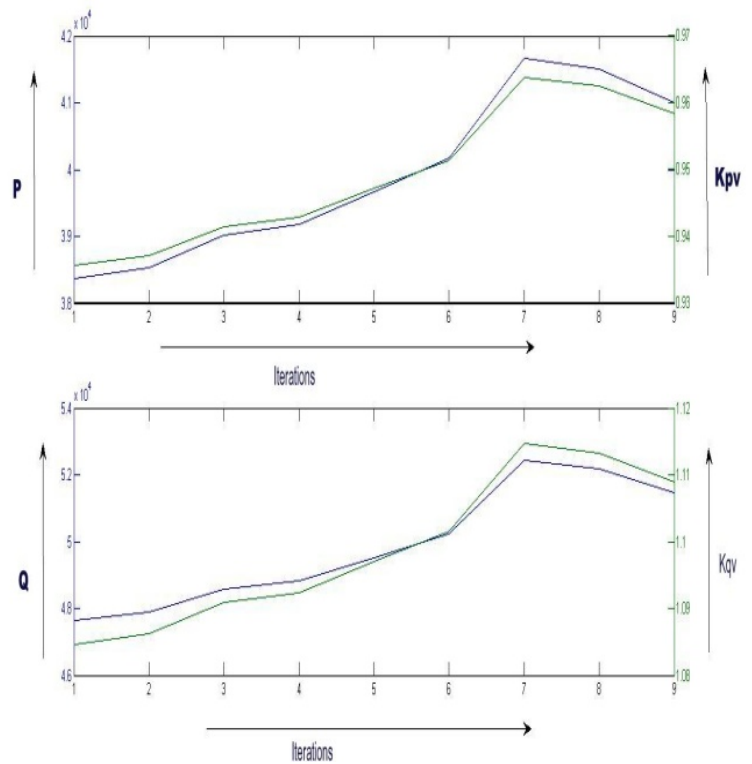

In the above graph we have shown the variation of $\mathrm{Kpv}$ and Composite load when there is a change in the Voltage.

\section{iii. Variation in Proportional Value}

In this case we have shown variation in the fractional value changes there is no change in the power and voltage is also kept constant and there are different fractional value i.e. the value of $P_{1}, P_{2}, P_{3}$ are varying and their sum is always equal to unity ,due to this variation their is change in the value of $\mathrm{Kpv}$ for Active Power and Kqv for Reactive Power due to which there is a change in the characteristic load at that node. This variation can be shown with the help of a graph which is drawn with the the help of Matlab software.
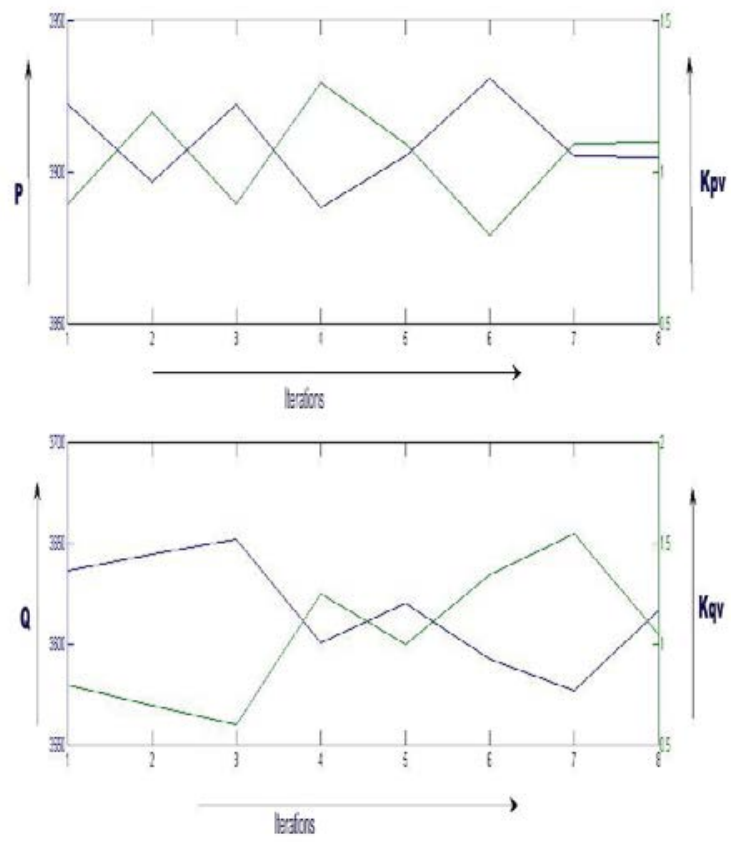

In the above graph we have shown the variation of Kpv and Composite load when there is a change in the Fractional Value.

\section{Curve Fitting of Above Variations}

- Variation of Voltage Profile and its quadratic equation obtained after curve fitting

- For Active Power(P)

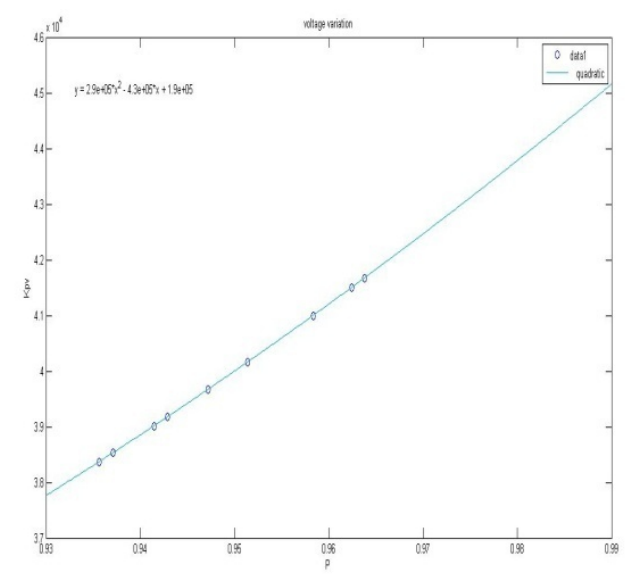

$y=2.9 e+05 * x^{2}-4.3 e+05 * x+1.9 e+5$

- For Reactive Power(Q)

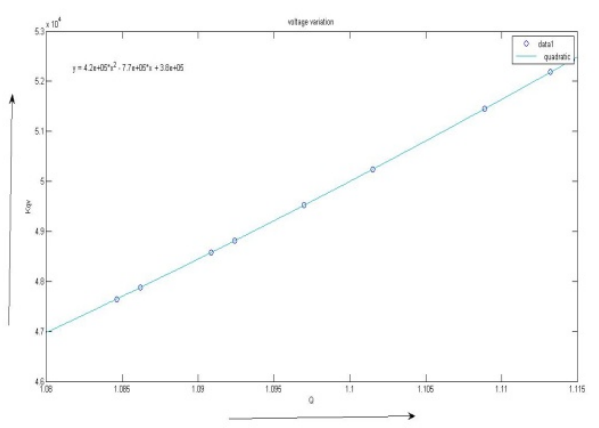

$y=4.2 e+05 * x^{2}-7.7 e+05 * x+3.8 e+5$

- Variation of Load Profile and its Quadratic Equation obtained after Curve fitting. 
- For Active Power(P)

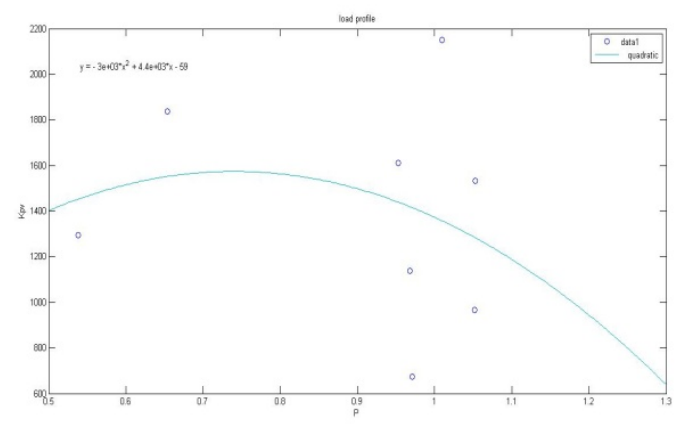

$$
y=-3 e+03 * x^{2}-4.4 e+03 * x-59
$$

- For Reactive Power

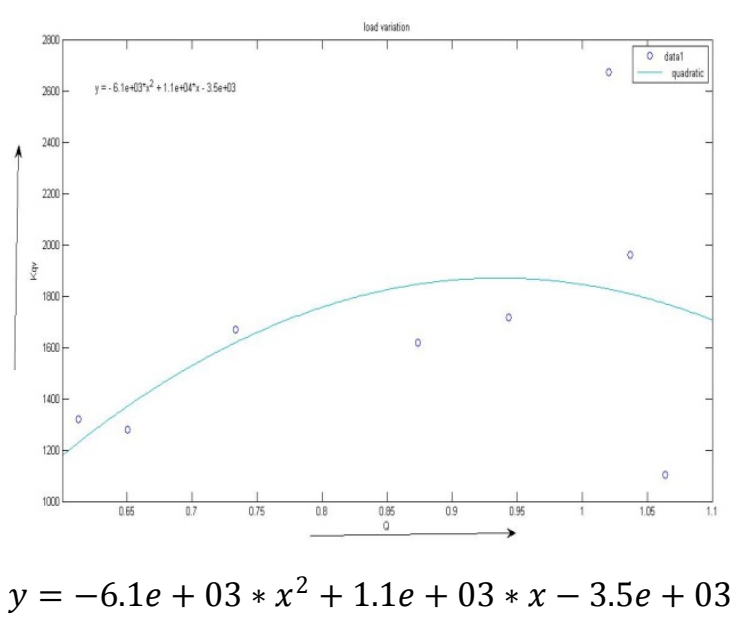

- Variation of Fractional Values and its Quadratic equation obtained after curve fitting.

\section{- For Active Power}

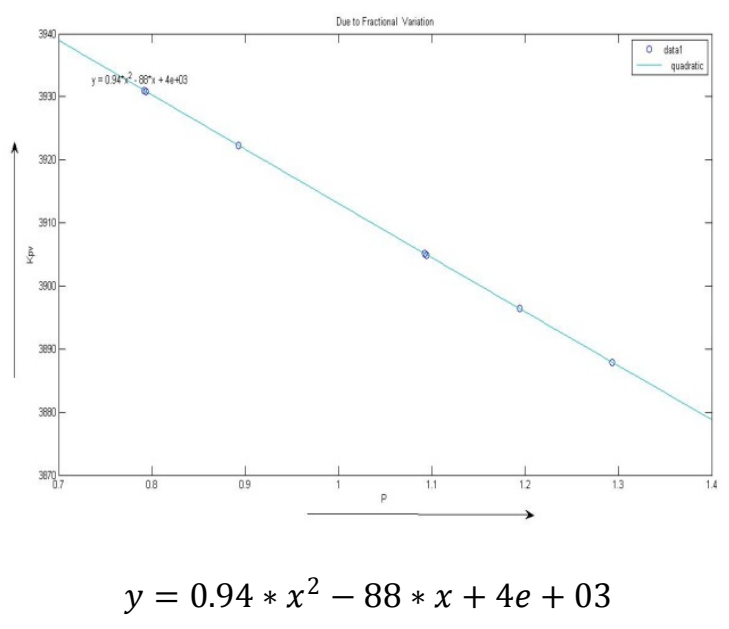

- For Reactive Power

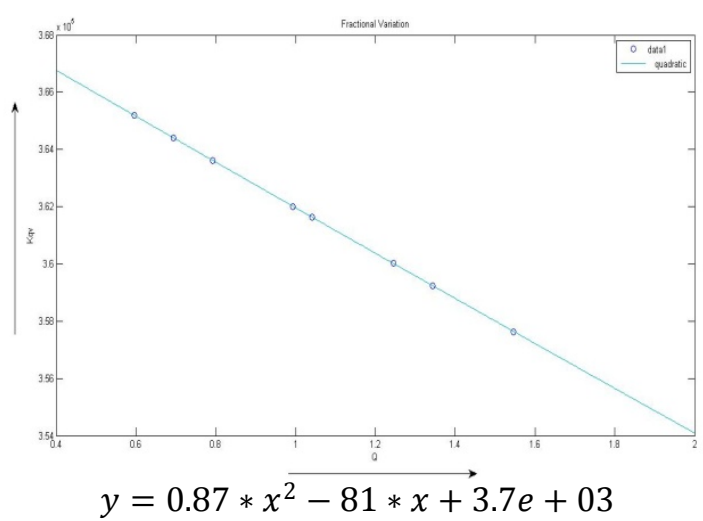

IV. CONCLUSION

Hence from the above P-V and Q-V curves drawn in the above mentioned three different cases we can see the variation of the Characteristic Load at a given node when there is change in the value of the power coefficients i.e. Kpv and Kqv which deals with active and reactive power respectively.

From the curve tracing in the above cases we can easily find out that how the value of load changes when there is a change in any of the profile. We can also draw out a conclusion from the quadratic equation which we have derived from the above drawn curves.So finally we can conclude that in any power system network when there are more than one Constant ZIP load at a particular node then there will be a change in the value of the power coefficients and due to the change in that value the characteristic load also changes.

\section{REFERENCES}

[1] Sabir, S.A.Y., and Lee, D.C.: 'Dynamic load models derived from data aquired during system transients', IEEE Trans. Power Syst., 1982, 101, (9), pp. 3365-3372

[2] Frantz, T., Gentile, T., Ihara, S., Simons, N., and Waldron, M.: 'Load behavior observed in LILCO and RG\&E systems', IEEE Trans. Power Syst., 1984, 103, (2), pp. 819-831

[3] Liang, Y., Nwankpa, C.O., Fischl, R., DeVito, A., and Readinger, S.C.: 'Dynamic reactive load model', IEEE Trans. Power Syst.,1998, 13, (4), pp. 1365-1372

[4] Lin, C.-J., Chen, Y.-T., and Chiou, C.-Y., et al.: 'Dynamic load models in power systems using the measurement approach', IEEE Trans. Power Syst., 1993, 8, (1), pp. 309-315

[5] Chiang, H.D., Wang, J.-C., Huang, C.-T., Chen, Y.-T., and Huang, C.H.: 'Development of a dynamic ZIP-motor load model from on-line field measurements', Int. J. Electr. Power Energy Syst., 1997, 19, (7), pp. 459-468

[6] IEEE Task Force on Load Representation for Dynamic Performance: 'Bibliography on load models for power flow and dynamic performance simulation', IEEE Trans. Power Syst., 1995, 10, (1), pp. 523-538

[7] IEEE Task Force on Load Representation for Dynamic Performance: 'Load representation for dynamic performance analysis', IEEE Trans. Power Syst., 1993, 8, (2), pp. 472-482

[8] IEEE Task Force on Load Representation for Dynamic Performance: 'Standard load models for power flow and dynamic performance simulation', IEEE Trans. Power Syst., 1995, 10, (3), pp. 1302-1313

[9] Concordia, C., and Ihara, S.: 'Load representation in power system stability studies', IEEE Trans. Power Syst., 1982, 101, (4), pp. 969-977

[10] Taylor, C.: 'Power system voltage stability’ (McGraw-Hill, 1994)

[11] Hajagos, L., and Danai, B.: 'Laboratory measurements and models of modern loads and their effect on voltage stability studies', IEEE Trans. Power Syst., 1998, 13, (2), pp. 584-592 
[12] Korunovic', L., and Stojanovic', D.: 'Load model parameters on low and middle voltage in distribution networks', Elektroprivreda,2002, (2), pp. 46-56

[13] Ribeiro, J., and Lange, F.: 'A new aggregation method for determining composite load characteristics', IEEE Trans. Power Syst., 1982, 101, (3), pp. 2869-2875

[14] Price, W., Wirgau, K., Murdoch, A., Mitsche, J., Vaahedi, E., and ElKady, M.: 'Load modeling for power flow and transient stability computer studies', IEEE Trans. Power Syst., 1988, 1, (4), pp. 180-187

[15] Milanovic', J.: 'On unreliability of exponential load models', Electr.Power Syst. Res., 1999, 49, (1), pp. 1-9

[16] Hill, D.: 'Nonlinear dynamic loads in power systems with recovery for voltage stability studies', IEEE Trans. Power Syst., 1993, 8, (1), pp. 166-176

[17] Karlsson, D., and Hill, D.: 'Modelling and identification of nonlinear dynamic loads in power systems', IEEE Trans. Power Syst., 9, (1), pp. 157-166

[18] Knyazkin, V., Cañizares, C., and So“der, L.: 'On the parameter estimation and modeling of aggregate power system loads', IEEE Trans. Power Syst., 2004, 19, (2), pp. 1023-1031

[19] Kundur, P.: 'Power system stability and control' (McGraw-Hill, 1994)

[20] Baghzouz, Y., and Quist, C.: 'Determination of static load models from LTC and capacitor switching tests'. Proc. IEEE PES Summer Meeting, July 2000, pp. 389-394

[21] Korunovic', L., and Stojanovic', D.: 'Load modeling in distribution networks', Facta Universitatis (Nis`), Ser. Elec. Energ., 2002, 15, (3), pp. 419-427

[22] Xu, W., and Mansour, Y.: 'Voltage stability analysis using generic dynamic load models', IEEE Trans. Power Syst., 1994, 9, (1), pp. 479493

[23] Nelles, O.: 'Nonlinear system identification: from classical approaches to neural networks and fuzzy models' (Springer, 2000)

[24] Korunovic', L., and Stojanovic', D.: 'Determination of dynamic load model parameters'. Proc. 2nd Regional Conf. and Exhibition on Electricity Distribution, October 2006, Zlatibor, Serbia), vol. R-6.6

[25] Merkle, M.: 'Probability and statistics for engineers and students of technical sciences' (Akademska misao, 2006, 2nd edn.) 